\title{
XIX. \\ Beschreibung einer Difformität des Thorax mit Defect der Rippen, nebst Bemerkungen über die Herzbewegung.
}

\author{
Von Dr. Frickhoeffer in Idstein (Nassau).
}

(Hierzu Taf. VI. Fig. 1.)

Die nifformitä des 14jälrigen Knaben, Sohn des Wagnormeister Blum dahier, ist angehoren und bestelt der Hauptsache nach in einer starken Kyphosis und Scoliosis, verbunden mit den davon abbängigen Verkrümmungen der Rippen und mit einem theilweisen Mangel derselben.

Die Verbiegung der Wirtelsäule beginnt in der Mitte der Halswirbel und geht zuerst von linlss nach rechts und hinten, dann fast quer und zuletzt strack von oben nach unten und einwärts, und bildet so ungefähr ein umgekehrtes lateini-

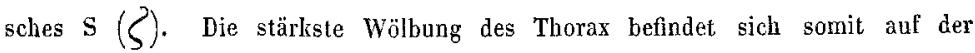
rechten Hälfte nach vorn, auf der linken nach hinten. Die rechte Seite ist einwärts, die linke auswärts gebogen und der grösste Querdurchmesser der Brust berülirt die Mitte des Brustbeins und den unteren Winkel des linken Schulterblatts.

Folgende Punkte sind an der vorderen Fläche des Thorax bemerkenswerts:

1. Das Sternum (siehe Zeichnung $h$ ) ist breiter als im normalen Zustand und verläuft schicf von oben und rechts nach links und unten.

2. Yon den Rippen linkerseits stelit nur die Iste in Verbindung mit dem Brusibein (c), die 2 te endigt $2 \frac{1}{2}$ Zoll von demselben, die 3te, 4te und 5te, indem sie sich von der Achselhöhle aus fast gerade herabstrecken, stehen $\mathbf{3}$ Zoll weit von dem Brustbein entfernt und die äbrigen, durch gemeinschaftliche Knorpel verbunden, bilden von links nach reclits einen halbmondförmigen Bogen und endigen $(g) 1 \frac{1}{2}$ Zoll weit von dem rudimentären Proc. ensif. (i).

3. Durch diese Missbildung und das Fehlen eines Theils der Rippen entsteht in der linken Brusthälfte ein annähernd gleichschenliliges Dreieck $(\boldsymbol{O})$, dessen Basis nach oben gekelrt ist und von der isten Rippe und dem anteren land des 


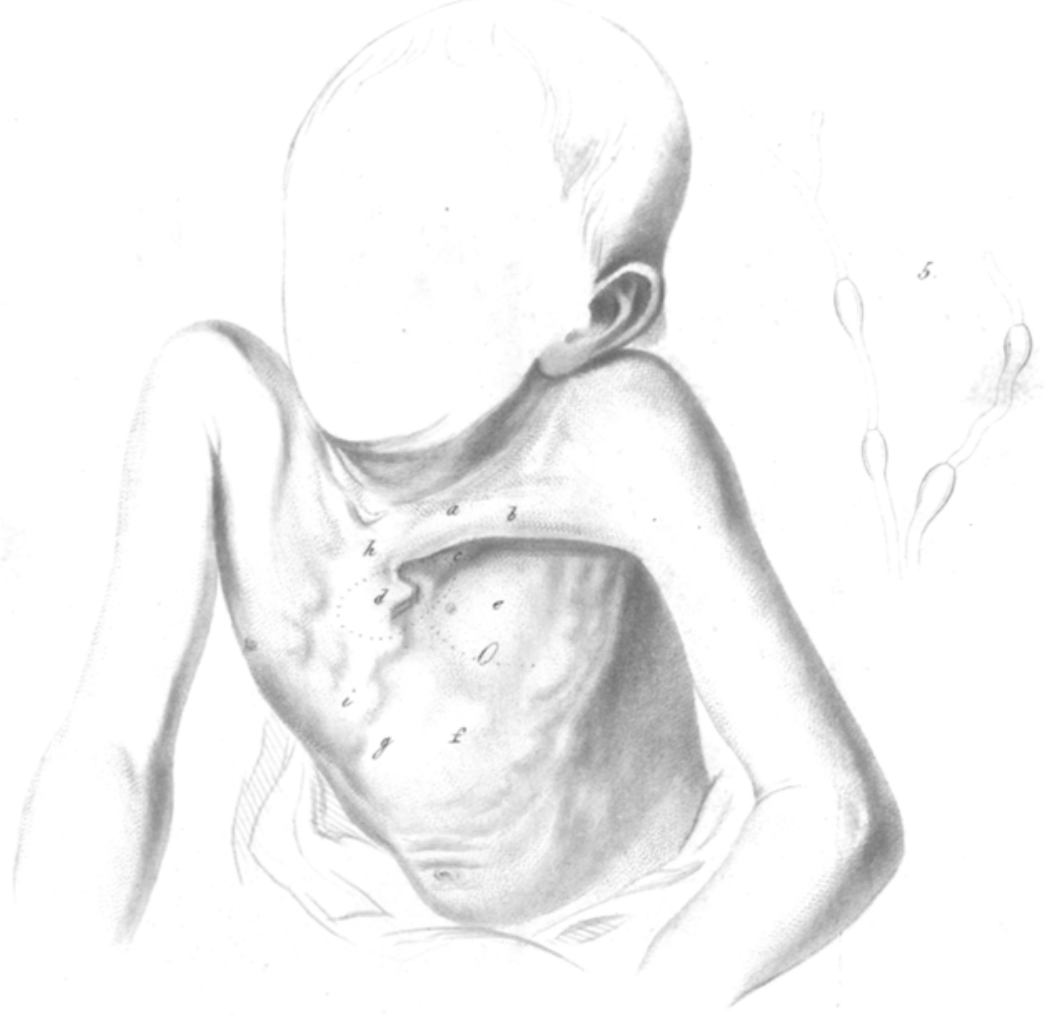

2 .

3.

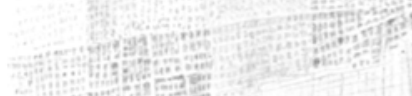

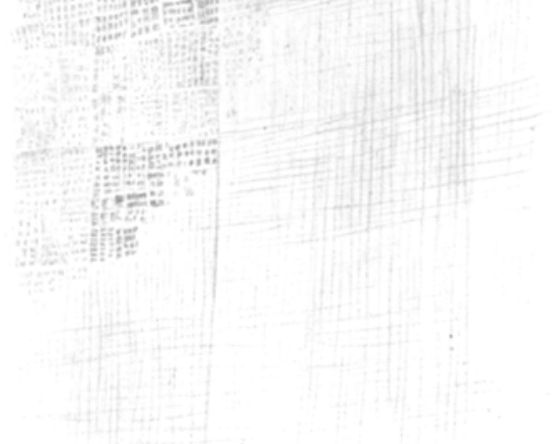

183. a vis?

398
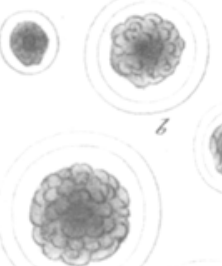

(4)

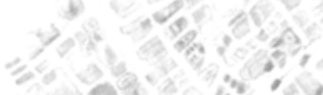

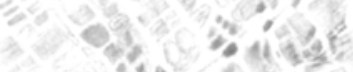

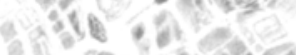

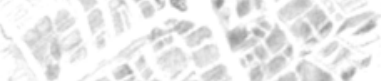

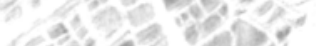

$693^{\circ}$ and

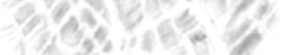

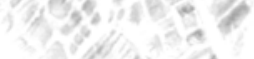

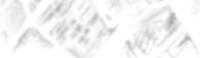

$(893) d$
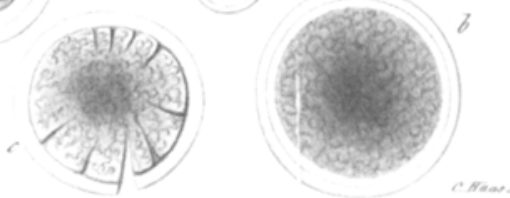
Musc. pect. major (b) gebildet wird, dessen abgestumpte Spitze aber nach unten liegt. Gegen seinen oberen rechten Winkel hin befindet sich die Brustwarze. Jeder Schenkel des Dreiecks ist ungefähr $3 \frac{1}{2}$ Zoll lang, seine Fläche liegt $\frac{1}{2}$ Zoll tiefer als die der übrigen Brustwand.

4. In diesem dreieckigen Raume sind die darunter gelegenen Theile der Brustorgane nur von der Haut bedechit und man sieht unter derselben deutlich die Bewegung eines Theils der linken Lunge und des Herzens.

5. Die Bewcgungen des Herzens beobachtet $\operatorname{man}$ in dem rechten oberen Winkel des beschriebenen Raumes; man unterscheidet dabei deullich eine Bewegung von links nach rechts und füllt bei der Palpation unmituelbar unter der Haut den Herzstoss, der aber in zwei Zeitmomente fällt und dadurch als ein doppelter erscheint, als ein längerer stärkerer und ein kürzerer schwächerer. Der erste ist synebronisch mit der Systole, der zweite folgt dem ersten und der Systole als schneller schwächerer Nachschlag *).

Die Bewegung der Lunge erkennt_man dentlich an einem mit dem Ein- und Ausathmen synchronischen $\mathrm{Ab}$ - und Aufsteigen and an einem von links nach rechts sichtbaren Hin - und Herrutsehen des dem Herzen zugekehrten Lungenrandes.

6. Aus den Ergebnissen der Percussion gelit hervor, dass das Herz von der beschriebenen pulsirenden Stelle an nach rechts unter dem Brustbein hín und nach unten gegen den Schwertfortsatz hin sich erstreclit, dass es somit eine mehr quere von links und binten nach rechts, vorn und unten gerichtete Lage hat $(d)$. Es wird hierdurch wahrscheinlich, dass der in dem Dreieck pulsirende Theil des Herzens einem Theil des Jinken Ventrikels entspricht.

Ferner ergibt die lercussion in dem linken oberen Winkel des Dreiecks einen sonoren Ton in Umkrejs von 2 Quadratzoll, der somit einen Theil der linken Lunge bedeckt und den grössten Raum des Dreiecks einnimmt (e): Das übrige untere Drittheil liefert einen vollständig leeren Ton und müsste den angefüllten Magen und linken Leberlappen enthatten.

7. Die Auscultation ergibt an dem freiliegenden Theil des Herzens einen vollständig normalen 1 sten und 2ten Ton, von welchen der erste der längere ist and den Accent hat. An der ülsrigen, der Lage des Herzens entsprechenden Gegend sind ebenfalls diese beiden Töne in gleicher Art, nur der Entfernung gemäss schwächer, hörbar; der Accent liegt stets auf dem crsten längeren Ton. Der 1 ste lảngere Ton an der freiliegenden Herzpartie entspricht zeitlich dem oben beschricbenen stärkeren Stoss und der 2te kïrzere dem schwächeren und kürzeren Stoss.

In der Gegend, wo zufolge des sonoren Tones ein Theil der linken Lnnge Jiegt, ist einfaches vesiculäres Athmen hörbar. An der Stelle des leeren Tones im unteren Drittel des Dreiecks ist Nichts zu bören.

*) In der Zeichnung crsclieint die Fläche, wo dic Herzhewegung sichtbar ist, kleiner, als in der Natur, weil durch den Winkel, wnter dem síe sich von dem Brustheiniand abdacht, ein bedentender Schatten in der Zeichnung cutsteht. 
Gehen wir auf die Beschreibung dieses Falles etwas näher ein, so ist vor Allem nicht zu verkennen, dafs derselbe manches der Fissura sterni congenita des A. Groux Analoge besitzt, die in der medicinischen Welt so grofses Aufsehen erregt und die Percuttoren und Auscultatoren ersten Ranges zu den widersprechendsten Resultaten geführt hat. Auch hier liegt ein Theil des Herzens, nur von der Haut und dem Pericardium bedeckt, frei vor unserem Auge, aufserdem ein Theil der linken Lunge, des Magens und des linken Leberlappens, aber nicht hervorgetreten aus einer Spalte des Sternum, sondern aus einem durch rudimentäre Bildung der Rippen bedingten Defect der linken Thoraxwand von ganz beträchtlicher Gröfse.

Ist diese Anomalie an und für sich schon von grofsem Interesse, so wird sie es in noch höherem Grade durch die Möglichkeit einer directeren Beobachtung der Herzbewegung, die, wenn auch micht zu neuen, doch zu solchen Resultaten führt, dafs dadurch die eine oder andere hier und da bestrittene Behauptung eine weitere Stuitze erhalten oder verlieren kann.

Zunächst hahdelt es sich um die Frage, welcher Theil des Herzens ist es, der in dem oberen rechten Winkel des beschriebenen Dreiecks gesehen und gefühlt wird?

Berücksichtigt man, dafs, bedingt durch die Deformität des Brustkastens, vermöge deren die rechte Hälfte in ihrem Raume beeinträchtigt und der ganze Brustkorb von oben nach unten und hinten comprimirt ist, sämmtliche Eingeweide mehr nach links und hinten verschoben sein müssen, dafs das Dreieck um einen halben Zoll tiefer liegt, als die übrige Brustwand; berücksichtigt man, dafs das Herz, um mit dem Bogen der Aorta und ihrem absteigenden Theil der Krümmung der Wirbelsäule folgen zu können, höher nach oben und zugleich mehr horizontal als vertical gelegen sein muls; nimmt man hinzu die Ergebnisse der Percussion, welche eine mehr quere von links und hinten nach rechts, vorn und unten gehende Lage andeuten, ferner die Resultate der Auscultation, welche an dem frei liegenden Theile zwei normale Herztöne, und zwar hier an 
deutlichsten, hören lassen, von denen der erste der längere ist und den Accent hat, nach Skoda das Kennzeichen für die in den Kammern entspringenden Töne, während es sich bei den Tönen in den Arterien umgekehrt verbält, so unterliegt es wohl keinem Zweifel, dafs der zu Tage liegende Herzlheil der linken Kammer und zwar aller Wahrscheinlichkeit nach ihrem oberen seitlichen Drittheil angehört. Ueberhaupl konnle der Lage nach nur darüber ein Zweifel obwalten, ob man einen Theil der linken Kammer oder einen Theil der grofsen Gefälsstämme vor sich habe, worïber mir die Art der Töne in der bezeichneten Weise entschieden zu haben scheint.

2. Es ist dieser Fall, resp. das eclatante Fühlbarsein des Herzstofses an der frei liegenden Parlie der linken Kammer eine weitere Stülze für die Ansicht von Kiwisch, dafs der fïhlbare Herzstofs nicht von einem eigentlichen Stolse der Herzspitze gegen die Brustwand, sondern blos von dem Fühlbarwerden der systolischen Erhärtung des sich in die Intercostalräume hineinpressenden Herzmuskels herriihre. Denn in unserem Falle ist nur an der entsprechenden Stelle des linken Ventrikels in Folge seiner systolischen Erhärtung, sonst nirgends an der ganzen Brustwand der Stofs des Herzens fühlbar, und die Gründe, die Bamberger (Virchow's Archiv, Bd.IX. Hft. 3. S. 333.) für den im normalen Zustand nur an der Herzspitze bemerkbaren Stofs angibt, erhalten auch durch unsere Beobachtung volle Bestätigung. Er führt als Hindernisse des Fühlbarwerdens der systolischen Erhärtung der Ventrikel im Normalzusiande an: die geringere Dicke des rechten Ventrikels, der zumeist und zunächst der Brustwaud anliegt, das nähere Aneinanderstehen der oberen Rippen, die dickeren Brustmuskeln nach oben hin, und erklärt besonders noch: ,ich habe mich oft genug überzeugt, dafs bei Kindern und sehr abgemagerten Personen der Herzstofs häufig einen bei Weitem grörseren Raum einnimmt, ja in der That oft an allen Stellen deutlich gefühlt wird, an denen das Herz anliegt. Präparirt man bei einem Kaninchen die Brustmuskeln weg und legt die Intercostalräume blofs, so 
fúhlt man an jeder Stelle, wo man das Herz anliegen sieht, den Herzstofs gleich deutlich, wenn auch friher davon Nichts wahrzunehmen war."

In dem oben beschriebenen Falle fehlen jene Hindernisse und defshalb ist die normale systolische Contraction der Kammer und der dadurch bedingte Herzstofs fühlbar, und es geht daraus hervor, dals seine Ursache nicht in dem hebelartigen Anschlagen der Herzspitze, sondern in der plötzlichen Contraction und Erhärtung beider Kammern begründet ist.

3. Wie oben schon erwähnt, war an der freiliegenden Herzpartie ein in zwei Zeitmomente gelheilter Herzstofs bemerkbar, ein längerer stärkerer und ein schwächerer kürzerer, der ersle synchronisch mit der Systole, der zweite dem ersten als schneller schwächerer Nachschlag folgend, der erste ferner entsprechend dem ersten Herzton, der zweite dem zweiten Herzton. Diese Beobachtung war so eclatant, die Erschütterung durch den zweiten Stofs an dem aufgesetzten Finger so deutlich fühlbar, dafs an eine Täuschung nicht zu denken war, und zwei mit mir untersuchende Mediciner sich sofort von derselben Erscheinung überzeugten.

Zur Beurtheilung derselben ist es nothwendig, vorauszuschicken, dafs eine Krankheit des Herzens, eine Erweiterung oder Hypertrophie nirgends sich documentirte, dafs es also keine Anwendung auf diesen Fall finden kann, wenn Skoda (Perc. u. Ausc. Aufl. 3. S. 152.) hinsichtlich des doppelten Herzstofses sagt: ,in manchen Fällen mit Hypertrophie und Dilatation beider Ventrikel verursacht die Systole den Stols, die Diastole hebt die Brustwand gar nicht, aber man fühlt eine Erschülterung, und diesè ist nicht durch das Anschlagen des Herzens gegen die Brustwand hervorgebracht, sondern scheint gleichsarn von dem Zurücksinken dieses Organs gegen die Wirbelsäule herzurihhren. Diese Erscheinung ist offenbar dieselbe, welche Laennec den Stofs der Vorhöfe nannte."

Der hier beobachtete 2te Stofs scheint mir vielmehr ein und dieselbe Ursache zu haben, wie der 2te Ton, mit dem er synchronisch ist, nämlich den Stofs der in den Arterien 
enthaltenen Blutsäule gegen die Semilunarklappen nach der Systole der Kammern, und diels zwar aus folgenden Gründen:

a) Er findet statt in einem Zeitmoment, welches dem Ende der Systole entspricht, er folgt dem ersten systolischen Schock als leichter Nachschlag und stimmt also zeitlich überein mit der Füllung der Arterien und dem Schlufs der Semilunarklappen. Und dafs durch den Stofs der Arterienblutsäule gegen diese Klappen eine dem ganzen Herzen, vorzüglich aber dem den Arterien zunächst liegenden Theile mittheilbare Erschütterung möglich sei, dürfte keinem gegrïndeten $Z$ weifel unterliegen. Sie ist nur zu unbedeutend, um bei normalen Brustwandungen stets gefühlt zu werden.

b) Ich war durch die vorausgegangene Untersuchung zu dem Resultat gelangt, dals der freiliegende Herztheil der oberen seitlichen Partie des linken Ventrikels entspreche. Der in dieser Gegend hörbare zweite Ton konnte also auch der Thätigkeit des Ventrikels seine Entstehung verdanken, mithin auch der fragliche zweite Stofs in diesem entspringen. Eine solche Möglichkeit wird aber widerlegt durch den Ausspruch Skoda's 1. c. S. 187.: "man kann nicht behaupten, dafs bei normaler Beschaffenheit des Herzens der zweite Ton in den Ventrikeln immer gebildet wird, indem es häufig wahrscheinlich, nicht selten auch gewils ist, dafs der zweite Ton, den man über dem Herzen hört, in den Arterien entsteht und wegen seiner Intensität auch in einiger Entfernung vernehmbar ist. Aber es gibt gewils Fälle, wo man genöthigt ist, die Entstehung des 2ten Tones in der Gegend der Ventrikel selbst zuzugestehen. Es sind diefs solche, wo man den zweiten Ton über der Basis des Herzens fast gar nicht, oder nur sehr schwach, dagegen an der Herzspitze sehr laut und hell vernimm l."

In unserem Falle aber wird der zweite Ton nicht an der. Herzspitze, sondern gerade nach der Basis hin laut und hell vernommen, und es beweist diefs also, dafs er an den Semilunarklappen entstanden und von da weiler fortgeleitet ist. 
Der mit ihm synchronische zweite Herzstofs muls also aller Wahrscheinlichkeit nach an demselben Orte entspringen. Und zwar möchte hier die Annahme eines Rückstol'ses, nicht in dem Gutbrod-Skoda'schen Sinne, wie er für den systolischen Impuls geltend gemacht worden, gerechtfertigt erscheinen. Dieser Rückstofs entsteht dadurch, dafs während der beginnenden Diastole, also ehe die neue Blutwelle in die Arterie gelangt, das unmittelbar vorher in dieselbe getriebene Blut vermöge der Elasticität der Arterienwand gegen die nun sich aufblähenden und schliefsenden Semilunarklappen zurücksinkt; er könnte also gewissermaalsen als elastischer Rückstofs bezeichnet werden.

Wäre aber der zweite Ton als in der Kammer entstanden anzunehmen, d. h. wäre er an der Herzspitze besonders deutlich und nicht nach der Basis hin hörbar, so könnte auch die Ursache des zweiten Stofses in dem Anschlagen des Blutes gegen die Kammerwandungen während der Diastole gesucht werden, wenn nämlich die hypothetische Annahme Skoda's (l. c. S. 187.) allgemeine Gültigkeit besälse, dafs der Anschlag des Blutes gegen die Kammerwandungen während der Diastole der Ventrikel vielleicht zuweilen den zweilen Ton hervorbringe.

4. Was die vielbesprochenen hebelartigen Bewegungen des Herzens betriff, durch die früher Hope den Herzstofs erklärte, für welche nachher Volkmann in die Schranken trat, deren Existenz aber die neueren Untersuchungen von Bamberger und Kölliker (Virchow's Arch. Bd. IX. Hft.3.) wenigstens in ihrem ursächlichen Zusammenhang mit dem Herzstofs im höchsten Grade zweifelhaft machen, so ist unser Fall meines Erachtens nicht geeignet, ein besonderes Licht darüber zu verbreiten, weil ein zu kleiner Theil und zwar nur der Basis des Herzens der Beobachlung zugänglich ist und aufserdem die übrigen anatomischen Verhältnisse des Brustbaues zu sehr von der Norm abweichen, um ein unbestreitbares Urtheil zu gestatten. Nur soviel kann aus den Ergebnissen der Untersuchung geschlossen werden, dals dic Annahme einer zwei- 
armigen Hebelkraft nicht plausibel erscheint, weil in diesem Falle ein Zurücksinken der oberen Herzpartie, also auch des hier sichtbaren Theiles der linken Herzkammer mit der sie bedeckenden Brustwand während der Systole und während des dabei nothwendig erfolgenden Anschlags der Herzspitze gegen die Brustwand beobachtet werden mïste, wovon gerade das Gegentheil, nämlich ein Hervortreten und Hervorwölben des fraglichen Theiles bemerkbar ist.

5. Auch hinsichtlich der Locomolion des Herzens liefert unsere Beobachtung einiges Material. Die an dem zjemlich ausgebreiteten rippenlosen Theile der Brustwand stattindenden Bewegungen der unterliegenden Organe erforderten zu ihrer Würdigung eine angèstrengte Aufmerksamkeit, weil Herz- und Lungenbewegung ein beständiges Spiel der bedeckenden Haut verursachten. Bei längerer Prüfung aber stellte sich deutlich ein mit dem Ein- und Ausathmen synchronisches $A b$ - und Aufsteigen der Lunge mit einer geringen seitlichen Dislocation des Lungenrandes nach rechts heraus und eine mit der Systole gleichzeitige wellenförnige Bewegung des Herzens von links und oben nach rechts und unten. Augenscheinlicher noch wurde letztere Bewegung, wenn man auf der freien Herzfläche eine Querlinie mit Tinte punctirte. Diese Locomotion entspricht, wie man sich erinnern wird, der angegebenen Lage des Herzens, resp. seiner Längenaxe, und wird aller Wahrscheinlichkeit nach nicht hervorgerufen durch die Contraction und Verkürzung der Kammern in ihretn Längendurchmesser, eine als Ursache der. Locomolion nach unlen schon längst zurückgewiesene Annahme, sondern durch die Verlängerung der Blutsäule während der Systole, oder, wie Bambergex I. c. sich ausdrückt, durch die Längsdehnung der grofsen Gefäfsstämme, wie sie letzterer durch seine mit Kölliker vorgenommenen Vivisectionen in augenfälliger Weise dargethan hat. Sie konnte aber in unserem Falle gemäfs der Lage des Herzens nicht von oben nach unten, sondern mufste schief von links und oben nach rechts und unten erfolgen.

Ceber die sogenanile Rolation des Herzens um seine 
Queraxe konnte ich in der beschriebenen Abnormität keine Anhaltspunkte finden.

Diefs sind äbersichtlich die Beobachtungen, die mir ein so seltener Fall von Mifsbildung lieferte. Ich übergebe sje der Oeffentlichkeit mit der Ueberzeugung, dafs ein umsichtigerer und geäbterer Beobachter ihre Zahl und ihren Werth um Vieles hätte erhöhen können.

\section{Erklärung der Abbildung.}

Fig. 1. a. Sc̣hlüsselbein.

b. Pectoralis major.

c. 1 ste Rippe.

d. Ungefähre Lage des Herzens, nach den Ergebnissen der physikalischen Untersuchung mit Punkten umschrieben.

e. Linke Lunge.

f. Bogen der vereinigten Rippen.

g. Freier Raum zwischen Rippenknorpeln und Rroc. ensiformis.

h. Brustbein.

i. Proc. ensiformis.

o. Der nach rechts yon dem Brusthein, nach ohen von dem pect. major, nach links und unten von den Rippen begrenzte, dreieckige, rippenlose Raum. 